\title{
THE KNESER PROPERTY FOR ABSTRACT RETARDED FUNCTIONAL DIFFERENTIAL EQUATIONS WITH INFINITE DELAY
}

\author{
HERNÁN R. HENRÍQUEZ
}

\author{
Received 18 June 2002
}

\begin{abstract}
We establish existence of mild solutions for a class of semilinear first-order abstract retarded functional differential equations (ARFDEs) with infinite delay and we prove that the set consisting of mild solutions for this problem is connected in the space of continuous functions.
\end{abstract}

2000 Mathematics Subject Classification: 34K30, 34G20, 47D06.

1. Introduction. The purpose of this paper is to establish existence of mild solutions of a semilinear abstract retarded functional differential equation (ARFDE) with infinite delay of first order, and to show that under general conditions the set formed by the mild solutions is connected in the space of continuous functions. This property is known in the literature as the Kneser's property. We refer to [5] for the original result in the frame of differential equations and to [9] for a similar result for functional equations.

We start with an abstract statement of this property. In this statement, we denote by $V_{\delta}(B)$ the $\delta$-neighborhood of a set $B$ in a metric space.

LEMmA 1.1. Let $X$ and $Y$ be metric spaces, $B$ a closed subset of $Y$, and $T: X \rightarrow$ $Y$ a continuous function. Let $S=T^{-1}(B)$ and assume that there is a compact set $K \subseteq X$ such that for each $\varepsilon>0$, there is a set $K_{\varepsilon} \subseteq K$ with the following properties:

(i) the sets $K_{\varepsilon}$ are connected;

(ii) $d\left(x, K_{\varepsilon}\right)<\varepsilon$ for all $x \in S$;

(iii) $T\left(K_{\varepsilon}\right) \subseteq V_{\delta(\varepsilon)}(B)$, where $\delta(\varepsilon) \rightarrow 0$, as $\varepsilon \rightarrow 0$.

Then $S$ is connected.

Proof. We assume that $S$ is not connected. We derive the existence of nonempty disjoint closed sets $F_{1}$ and $F_{2}$ such that $S=F_{1} \cup F_{2}$. This implies that both $F_{1}$ and $F_{2}$ are closed in $X$ and $d\left(F_{1}, F_{2}\right)=\eta>0$. Let $U$ be the $\eta / 2$ neighborhood of $F_{1}$. It is clear that for each $0<\varepsilon<\eta / 2$, the sets $K_{\varepsilon} \cap U \neq \Phi$ and $K_{\varepsilon} \cap(X \backslash \bar{U}) \neq \Phi$. Since $K_{\varepsilon}$ is connected, it follows that $\operatorname{Fr}(U) \cap K_{\varepsilon} \neq \Phi$. Thus, we can choose $x_{n} \in \operatorname{Fr}(U) \cap K_{n}$, where $K_{n}=K_{\varepsilon}$, for $\varepsilon=1 / n$ with $n \in \mathbb{N}$ and $n \geq N$. Since $K_{n} \subseteq K$, there is a subsequence, which we denote by the same index, such that $x_{n} \rightarrow x$, as $n \rightarrow \infty$. Clearly, $x \in \operatorname{Fr}(U)$ and, in view of that $T$ is continuous, $T\left(x_{n}\right) \rightarrow T(x), n \rightarrow \infty$. Since $d\left(T\left(x_{n}\right), B\right) \rightarrow 0, n \rightarrow \infty$, it follows that 
$d(T(x), B)=0$, which in turn implies that $T(x) \in B$ and $x \in S$. Consequently, $x \in \operatorname{Fr}(U) \cap S$, which is absurd by the construction of $U$.

To apply this result to the set formed by the solutions of a certain equation, the following version is more convenient. Afterwards $X$ denotes a Banach space, $I \subseteq \mathbb{R}$ is a compact interval, and $C(I ; X)$ stands for the space of continuous functions from $I$ into $X$ endowed with the norm of uniform convergence.

COROLlary 1.2. Let $\mathcal{T}: C(I ; X) \rightarrow C(I ; X)$ be continuous and $S$ the set of fixed points of $\mathscr{T}$. Assume that there is a compact set $K \subseteq C(I ; X)$ such that for each $\varepsilon>0$, there is a set $K_{\varepsilon} \subseteq K$ with the following properties:

(i) the sets $K_{\varepsilon}$ are connected;

(ii) $d\left(x, K_{\varepsilon}\right)<\varepsilon$ for all $x \in S$;

(iii) $\|y-\mathscr{T} y\|_{\infty}<\delta(\varepsilon)$ for all $y \in K_{\varepsilon}$, where $\delta(\varepsilon) \rightarrow 0$, as $\varepsilon \rightarrow 0$.

Then $S$ is connected.

Another abstract version of the Kneser property, which is known as the Krasnoselskii-Perov theorem (see [13]), is obtained using degree theory.

Let $X$ be a Banach space endowed with a norm $\|\cdot\|$. Henceforth, we always assume that $A: D(A) \rightarrow X$ is the infinitesimal generator of a strongly continuous semigroup of linear operators $T(t)$ defined on $X$. In this paper, we are concerned with the initial value problem defined by the semilinear ARFDE with infinite delay

$$
x^{\prime}(t)=A x(t)+f\left(t, x_{t}\right), \quad 0 \leq t \leq \tau,
$$

with initial condition

$$
x_{0}=\varphi \in \mathscr{B},
$$

where $\mathscr{B}$ is a space, called the phase space for the equation, $f:[0, \tau] \times \mathscr{B}$ is an appropriated function, and $x_{t}$ represents the function defined from $(-\infty, 0]$ into $X$ by $x_{t}(\theta)=x(t+\theta),-\infty<\theta \leq 0$. Our purpose is to establish that the set of mild solutions of (1.1) and (1.2) is connected.

To study this problem in a general context, we will employ an axiomatic definition of the phase space $\mathscr{B}$ introduced by Hale and Kato [4]. To establish the axioms of space $\mathscr{B}$, we follow the terminology used in [8]. Thus, $\mathscr{B}$ will be a linear space of functions mapping $(-\infty, 0]$ into $X$ endowed with a seminorm $\|\cdot\|_{\Re}$. We will assume that $\mathscr{B}$ satisfies the following axioms.

(A) If $x:(-\infty, \sigma+a) \rightarrow X, a>0$, is continuous on $[\sigma, \sigma+a)$ and $x_{\sigma} \in \mathscr{B}$, then for every $t$ in $[\sigma, \sigma+a)$, the following conditions hold:

(i) $x_{t}$ is in $\mathscr{B}$;

(ii) $\|x(t)\| \leq H\left\|x_{t}\right\|_{\mathscr{B}}$;

(iii) $\left\|x_{t}\right\|_{\mathscr{B}} \leq K(t-\sigma) \sup \{\|x(s)\|: \sigma \leq s \leq t\}+M(t-\sigma)\left\|x_{\sigma}\right\|_{\mathscr{B}}$, where $H \geq 0$ is a constant; $K, M:[0, \infty) \rightarrow[0, \infty), K$ is continuous and $M$ is locally bounded and $H, K, M$ are independent of $x(\cdot)$. 
(A-1) For the function $x(\cdot)$ in $(A), x_{t}$ is a $\mathscr{B}$-valued continuous function on $[\sigma, \sigma+a)$.

(B) The space $\mathscr{B}$ is complete.

Throughout this paper, we always assume that $\mathscr{B}$ is a phase space.

2. Abstract retarded functional differential equations. Henceforth, $T(t)$ is a strongly continuous semigroup of linear operators on $X$ with infinitesimal generator $A$. We refer the reader to $[3,12]$ and the references cited therein for the theory of strongly continuous semigroup and the associated abstract Cauchy problem (ACP). We only mention here a few results needed for our developments.

The existence and regularity of solutions of the first-order ACP

$$
\begin{gathered}
x^{\prime}(t)=A x(t)+h(t), \quad t \geq 0, \\
x(0)=x_{0},
\end{gathered}
$$

where $h:[0, \infty) \rightarrow X$ is a locally integrable function, have been treated in several works (see $[3,12]$ ). We only recall here that the function

$$
x(t)=T(t) x_{0}+\int_{0}^{t} T(t-s) h(s) d s, \quad t \geq 0,
$$

is said to be a mild solution of (2.1).

Similarly, the existence of solutions of the semilinear ACP has been discussed in [1, 11]. Our interest in this section is to establish a Kneser's type property for the solutions of the functional semilinear first-order ACP (1.1) and (1.2). Next, we abbreviate the notation by writing $I=[0, \tau]$.

We begin with a result that ensures existence of solutions under quite general hypotheses which are suitable for our purposes. To study this initial value problem, we assume that the function $f: I \times \mathscr{B} \rightarrow X$ satisfies the following Carathéodory conditions:

(a) $f(t, \cdot): \mathscr{B} \rightarrow X$ is continuous a.e., $t \in I$;

(b) for each $\psi \in \mathscr{B}$, the function $f(\cdot, \psi): I \rightarrow X$ is strongly measurable.

In what follows, to study problem (1.1) and (1.2) and in order to avoid some cumbersome notations, if $x: I \rightarrow X$ is a function such that $x(0)=\varphi(0)$, we identify $x$ with its extension $x:(-\infty, \tau] \rightarrow X$ defined on $(-\infty, 0]$ by $x(\theta)=$ $\varphi(\theta)$. Furthermore, if $B \subseteq C(I ; X)$ is a set such that $x(0)=\varphi(0)$, for every $x \in$ $B$, we denote by $H(B) \subseteq C(I ; \mathscr{B})$ the set consisting of the continuous functions $t \rightarrow x_{t}$ and $x \in B$.

Expression (2.2) motivates the following concept of mild solution.

DEFINITION 2.1. We say that a function $x: I \rightarrow X$ is a mild solution of problem (1.1) and (1.2) if $x$ is a continuous function that satisfies the integral 
equation

$$
x(t)=T(t) \varphi(0)+\int_{0}^{t} T(t-s) f\left(s, x_{s}\right) d s, \quad 0 \leq t \leq \tau .
$$

In what follows, we denote by $B_{R}$ the closed ball with center at 0 and radius $R$ in an appropriated space. Since the strongly continuous semigroups are uniformly bounded on bounded intervals, we represent by $\widetilde{M}$ a constant such that $\|T(t)\| \leq \widetilde{M}$ for all $0 \leq t \leq \tau$. Moreover, we abbreviate our notations by writing $K=\sup _{0 \leq t \leq \tau} K(t)$ and $M=\sup _{0 \leq t \leq \tau} M(t)$ and we denote by $\overline{c o}(B)$ the closed convex hull of a set $B$.

THEOREM 2.2. Assume that the following conditions hold:

(H1) for each $R>0$, there is a positive integrable function $\gamma_{R} \in \mathscr{L}^{1}(I)$ such that $\sup \left\{\|f(t, \psi)\|:\|\psi\|_{\mathscr{B}} \leq R\right\} \leq \gamma_{R}(t)$ a.e., $t \in I$;

(H2) for each $0<t \leq \tau$ and $R \geq 0$, the set $\left\{T(t) f(s, \psi): 0 \leq s \leq \tau\right.$, $\left.\|\psi\|_{\mathscr{B}} \leq R\right\}$ is relatively compact;

(H3) $\liminf _{R \rightarrow \infty}(\widetilde{M} K / R)\left(\int_{0}^{T} \gamma_{R}(s) d s\right)<1$.

Then there is a mild solution of (1.1) and (1.2). In further, if the following condition is fulfilled:

(H4) $\limsup _{R \rightarrow \infty}(\widetilde{M} K / R)\left(\int_{0}^{T} \gamma_{R}(s) d s\right)<1$,

then the set $\mathscr{Y}$ formed by the mild solutions of (1.1) and (1.2) is compact in $C(I ; X)$ and the set $H(\mathscr{S})$ is compact in $C(I ; \Re)$.

Proof. Let $C_{\varphi}=\{x \in C(I ; X): x(0)=\varphi(0)\}$. We define the map $\mathscr{T}: C_{\varphi} \rightarrow$ $C_{\varphi}$ by

$$
\mathscr{T}(x)(t)=T(t) \varphi(0)+\int_{0}^{t} T(t-s) f\left(s, x_{s}\right) d s .
$$

Clearly, $\mathscr{T}$ is well defined and the Lebesgue's dominated convergence theorem implies that $\mathscr{T}$ is continuous. We affirm that there exists $n \in \mathbb{N}$ such that $\mathscr{T}$ : $B_{n} \rightarrow B_{n}$. In fact, if we assume that the assertion is false, we can select an increasing sequence $R_{j}$ such that

$$
\lim _{j \rightarrow \infty} \frac{1}{R_{j}} \int_{0}^{\tau} \gamma_{R_{j}}(s) d s=\alpha=\liminf _{R \rightarrow \infty} \frac{1}{R} \int_{0}^{\tau} \gamma_{R}(s) d s<1,
$$

as well as a sequence $\left(n_{j}\right)_{j}$ in $\mathbb{N}$ and a sequence $x^{j} \in B_{n_{j}}$ such that $q_{j}=$ $K n_{j}+M\|\varphi\|_{\mathscr{B}} \leq R_{j} \leq K\left(n_{j}+1\right)+M\|\varphi\|_{\Re}$ and $\left\|\mathscr{T}\left(x^{j}\right)\right\|>n_{j}$. Therefore, for each $t \in I$, we have that $\left\|x_{t}^{j}\right\|_{\mathscr{B}} \leq q_{j}$ from which it follows that

$$
n_{j}<\left\|\mathscr{T}\left(x^{j}\right)\right\| \leq \widetilde{M}\|\varphi(0)\|+\widetilde{M} \int_{0}^{\tau} \gamma_{R_{j}}(s) d s
$$


Hence, we obtain that

$$
\begin{aligned}
1 & <\frac{\widetilde{M}\|\varphi(0)\|}{n_{j}}+\frac{\widetilde{M}}{n_{j}} \int_{0}^{\tau} \gamma_{R_{j}}(s) d s \\
& =\frac{\widetilde{M}\|\varphi(0)\|}{n_{j}}+\widetilde{M} \frac{R_{j}}{n_{j}} \frac{1}{R_{j}} \int_{0}^{\tau} \gamma_{R_{j}}(s) d s \longrightarrow \widetilde{M} K \alpha, \quad j \longrightarrow \infty,
\end{aligned}
$$

which contradicts (H3).

Next, using (H1) and (H2), we establish that $\mathscr{T}$ is completely continuous. Since $\mathscr{T}$ is continuous, it only remains to prove that $\mathscr{T}$ takes bounded sets into relatively compact sets in the space $C(I ; X)$. From the Ascoli-Arzela theorem, it is sufficient to prove that for each $R \geq 0$, the set $\left\{\mathscr{T}_{0}(x)(t):\|x\|_{\infty} \leq R\right\}$ is relatively compact in $X$ for all $0 \leq t \leq \tau$ and that the set $\left\{\mathscr{T}_{0}(x):\|x\|_{\infty} \leq R\right\}$ is equicontinuous, where we have denoted

$$
\mathscr{T}_{0}(x)(t)=\int_{0}^{t} T(t-s) f\left(s, x_{s}\right) d s .
$$

We begin by establishing the first assertion. Let $t>0$ and we take $\varepsilon>0$ small enough. We can write

$$
\begin{aligned}
\mathscr{T}_{0}(x)(t)= & \int_{0}^{t-\varepsilon} T(t-s) f\left(s, x_{s}\right) d s+\int_{t-\varepsilon}^{t} T(t-s) f\left(s, x_{s}\right) d s \\
= & \int_{0}^{t-\varepsilon} T(t-\varepsilon-s) T(\varepsilon) f\left(s, x_{s}\right) d s \\
& +\int_{t-\varepsilon}^{t} T(t-s) f\left(s, x_{s}\right) d s .
\end{aligned}
$$

Applying the mean value theorem for the Bochner integral (see [10]), it follows that

$$
\begin{aligned}
\int_{0}^{t-\varepsilon} & T(t-\varepsilon-s) T(\varepsilon) f\left(s, x_{s}\right) d s \\
& \in(t-\varepsilon) \overline{\operatorname{CO}}\left\{T(t-\varepsilon-s) T(\varepsilon) f(s, \psi):\|\psi\|_{\mathscr{B}} \leq Q, 0 \leq s \leq t-\varepsilon\right\},
\end{aligned}
$$

where $Q=K R+M\|\varphi\|_{\mathscr{B}}$. By (H2), the set on the right-hand side is compact so that the first term on the right-hand side of (2.9) is included in a compact set which does not depend on the function $x(\cdot)$.

On the other hand, for the second term on the right-hand side of (2.9), we obtain the estimation

$$
\left\|\int_{t-\varepsilon}^{t} T(t-s) f\left(s, x_{s}\right) d s\right\| \leq \widetilde{M} \int_{t-\varepsilon}^{t} \gamma_{Q}(s) d s
$$

which shows that this term converges towards zero as $\varepsilon \rightarrow 0$ since $\gamma_{R^{\prime}}$ is integrable. Hence, we can assure that the set $V(t)=\mathscr{T}_{0}\left(B_{R}\right)(t)$ is relatively compact. 
To establish the second assertion, we first observe that

$$
\begin{aligned}
& \mathscr{T}_{0}(x)(t+h)-\mathscr{T}_{0}(x)(t) \\
&= \int_{0}^{t+h} T(t+h-s) f\left(s, x_{s}\right) d s-\int_{0}^{t} T(t-s) f\left(s, x_{s}\right) d s \\
&=(T(h)-I) \int_{0}^{t} T(t-s) f\left(s, x_{s}\right) d s \\
& \quad+\int_{t}^{t+h} T(t+h-s) f\left(s, x_{s}\right) d s .
\end{aligned}
$$

Note that the first term on the right-hand side is included in $(T(h)-I) V(t)$ so that this term converges to zero as $h \rightarrow 0$, independent of $x(\cdot) \in B_{R}$. Similarly, for the second term, we have

$$
\left\|\int_{t}^{t+h} T(t+h-s) f\left(s, x_{s}\right) d s\right\| \leq \widetilde{M} \int_{t}^{t+h} \gamma_{Q}(s) d s,
$$

which implies that this term tends towards zero as $h \rightarrow 0$.

Applying now the fixed-point theorem of Schauder, we infer that $\mathscr{T}$ has a fixed-point $x$ in $B_{n}$. Clearly, $x$ is a mild solution of (1.1) and (1.2). Moreover, the continuity of $\mathscr{T}$ implies that the set $\mathscr{S}$ consisting of mild solutions is closed.

On the other hand, if condition (H4) holds, then $\mathscr{S}$ is bounded. In fact, if we assume that $\mathscr{Y}$ is not bounded, then there exists a sequence of functions $x^{j} \in \mathscr{Y}$ such that $q_{j}=\left\|x^{j}\right\|_{\infty} \geq j$. Let $R_{j}=\sup _{0 \leq t \leq \tau}\left\|x_{t}^{j}\right\|_{\mathscr{B}} \leq K q_{j}+M\|\varphi\|$. Hence,

$$
\left\|x^{j}(t)\right\|=\left\|\mathcal{T} x^{j}(t)\right\| \leq \widetilde{M}\|\varphi(0)\|+\widetilde{M} \int_{0}^{T} \gamma_{R_{j}}(s) d s
$$

from which we obtain

$$
1 \leq \limsup _{j \rightarrow \infty} \widetilde{M} \frac{R_{j}}{q_{j}} \frac{1}{R_{j}} \int_{0}^{\tau} \gamma_{R_{j}}(s) d s \leq \widetilde{M} K \limsup _{R \rightarrow \infty} \frac{1}{R} \int_{0}^{\tau} \gamma_{R}(s) d s
$$

which is absurd by (H4). Finally, using that $\mathscr{T}$ is completely continuous, we deduce that $\mathscr{S}$ is compact. The last assertion is a direct consequence of the next property (see [8]). Thus, the proof is complete.

LEMMA 2.3. Let $\varphi \in \mathscr{B}$. If $B \subseteq C(I ; X)$ is a relatively compact set such that $x(0)=\varphi(0)$ for every $x \in B$, then $H(B)$ is relatively compact in $C(I ; \Re)$.

We refer to [6, 7] for another existence results.

Next, for future reference, we strengthen a property established in the proof of Theorem 2.2.

LEMmA 2.4. Assume that conditions (H1) and (H2) hold. Then for each $Q>0$, the set $\left\{\int_{0}^{t} T(t-s) f(s, y(s)) d s: 0 \leq t \leq \tau, y \in \mathscr{L}^{\infty}(I ; \mathscr{B}),\|y\|_{\infty} \leq Q\right\}$ is relatively compact in $X$. 
Proof. Proceeding as in the proof of Theorem 2.2, we infer that the set

$$
V(t)=\left\{\int_{0}^{t} T(t-s) f(s, y(s)) d s: y \in \mathscr{L}^{\infty}(I ; X),\|y\|_{\infty} \leq Q\right\}
$$

is relatively compact for each $0 \leq t \leq \tau$. We put $V=\bigcup_{0 \leq t \leq \tau} V(t)$ and we prove that this set is relatively compact. To this finality, we first observe that the following property holds: for each $\varepsilon>0$, there is $\delta>0$ such that

$$
V(t+h) \subseteq T(h) V(t)+B_{\varepsilon}, \quad|h| \leq \delta .
$$

In fact, we chose $\delta>0$ such that $\widetilde{M} \int_{t}^{t+h} \gamma_{Q}(s) d s \leq \varepsilon$ for $h \leq \delta$. Thus, for $x \in$ $V(t+h)$, we can write

$$
\begin{aligned}
x & =\int_{0}^{t+h} T(t+h-s) f(s, y(s)) d s \\
& =T(h) \int_{0}^{t} T(t-s) f(s, y(s)) d s+\int_{t}^{t+h} T(t+h-s) f(s, y(s)) d s .
\end{aligned}
$$

Since the first term on the right-hand side is included in $T(h) V(t)$ and the norm of the second term is less than or equal to $\widetilde{M} \int_{t}^{t+h} \gamma_{Q}(s) d s$, we establish the assertion.

Now, assuming that $\delta=\tau / n$ for some $n \in \mathbb{N}$ large enough, we have that

$$
V \subseteq \bigcup_{i=0}^{n-1}\left(\bigcup_{0 \leq h \leq \delta} T(h) V(i \delta)+B_{\varepsilon}\right)=\bigcup_{0 \leq h \leq \delta} T(h)\left(\bigcup_{i=0}^{n-1} V(i \delta)\right)+B_{\varepsilon}
$$

which implies that $V$ is relatively compact.

Next, we assume that problem (1.1) and (1.2) has mild solutions and we denote by $\mathscr{S}$ the set consisting of such solutions. For a set $\mathscr{F} \subseteq C(I ; Y)$, we denote by $\mathscr{F}(I)$ the set $\{f(t): f \in \mathscr{F}, t \in I\}$.

THEOREM 2.5. Assume that $f$ is a continuous function and that (H1) and (H2) are fulfilled. If in further the following conditions hold:

(H5) the set $\mathscr{Y}$ is compact,

(H6) $3 \widetilde{M} K \liminf _{R \rightarrow \infty}(1 / R) \int_{0}^{\tau} \gamma_{R}(s) d s<1$, then $\mathscr{S}$ is connected in $C(I ; X)$ and $H(\mathscr{Y})$ is connected in $C(I ; \Re)$.

PROoF. For the remainder of this proof, we abbreviate our notations by writing $h(t)=T(t) \varphi(0)$. On the other hand, applying (H5) and (H6), we can select a constant $R>0$ large enough such that $\|x\|_{\infty} \leq R$ for all $x \in \mathscr{Y}$ and

$$
\|h\|_{\infty}+3 \widetilde{M} \int_{0}^{\tau} \gamma_{Q}(s) d s \leq R,
$$


where $Q=K R+M\|\varphi\|$. This implies that

$$
K\|h\|_{\infty}+M\|\varphi\|+3 \widetilde{M} K \int_{0}^{\tau} \gamma_{Q}(s) d s \leq Q .
$$

Let $V$ be the set constructed in the proof of Lemma 2.4 for the constant $Q$. Without loss of generality, we can assume that $V$ is absolutely convex. We put $U=2 V$ and $U_{1}=3 V$. We denote $N_{1}=2 \widetilde{M} \int_{0}^{T} \gamma_{Q}(s) d s$.

We divide the proof into several steps.

STEP 1. For a division $d$ of $I$ formed by the points $0=t_{0}<t_{1} \cdots<t_{n-1}<$ $t_{n}=\tau$, we consider the function $z(\cdot) \in C_{\varphi}$ given by $z(0)=\varphi(0)$ and

$$
\begin{aligned}
z(t)= & h(t)+\sum_{k=1}^{i-1}\left(\int_{t_{k-1}}^{t_{k}} T(t-s) f\left(s, z_{t_{k-1}}\right) d s+\left(t_{k}-t_{k-1}\right) u_{k}\right) \\
& +\int_{t_{i-1}}^{t} T(t-s) f\left(s, z_{t_{i-1}}\right) d s+\left(t-t_{i-1}\right) u_{i}
\end{aligned}
$$

for $t_{i-1}<t \leq t_{i}$ and where we choose $u_{k}$ so that $\sum_{k=1}^{i}\left(t_{k}-t_{k-1}\right) u_{k} \in U$ and $\left\|\sum_{k=1}^{i}\left(t_{k}-t_{k-1}\right) u_{k}\right\| \leq N_{1}$, for all $i=1, \ldots, n$. Clearly, $z$ is a continuous function. Next, for a fixed $z$ given by (2.22), we denote by $\Psi(\cdot)$ and $u(\cdot)$ the step functions defined by $\Psi(0)=\varphi, u(0)=u_{1}, \Psi(t)=z_{t_{k-1}}$, and $u(t)=u_{k}$, for $t_{k-1}<t \leq t_{k}$ and $k=1, \ldots, n$. Thus, we can rewrite the definition of $z$ as

$$
z(t)=h(t)+\int_{0}^{t} T(t-s) f(s, \Psi(s)) d s+\int_{0}^{t} u(s) d s .
$$

We will show that $\|z(t)\| \leq R$ for $0 \leq t \leq \tau$, independent of the division $d$ and the choice of points $u_{i}$. In fact, from (2.20), we easily obtain that $\|z(t)\| \leq R$ for $0<t \leq t_{1}$. We assume that the assertion is true for $t \in\left[0, t_{i-1}\right]$ and we show that $\|z(t)\| \leq R$, for $t_{i-1}<t \leq t_{i}$. In fact, it is clear from the axioms of phase space that $\left\|z_{t}\right\|_{\mathscr{B}} \leq Q$ for $0 \leq t \leq t_{i-1}$, and since $\sum_{k=1}^{i-1}\left(t_{k}-t_{k-1}\right) u_{k}+\left(t-t_{i-1}\right) u_{i}$ is a convex combination of $\sum_{k=1}^{i-1}\left(t_{k}-t_{k-1}\right) u_{k}$ and $\sum_{k=1}^{i}\left(t_{k}-t_{k-1}\right) u_{k}$, from (2.23) we obtain

$$
\begin{aligned}
\|z(t)\| \leq & \|h(t)\|+\left\|\int_{0}^{t} T(t-s) f(s, \Psi(s)) d s\right\| \\
& +\left\|\sum_{k=1}^{i-1}\left(t_{k}-t_{k-1}\right) u_{k}+\left(t-t_{i-1}\right) u_{i}\right\| \\
\leq & \|h\|_{\infty}+\widetilde{M} \int_{0}^{\tau} \gamma_{Q}(s) d s+2 \widetilde{M} \int_{0}^{\tau} \gamma_{Q}(s) d s,
\end{aligned}
$$

which establishes our assertion.

STEP 2. To simplify the construction, we consider the points $t_{k}$ equally spaced with $\delta=t_{k}-t_{k-1}$ and, in addition to the conditions considered in 
Step 1, we suppose that

$$
\left\|\delta \sum_{k=i+1}^{j} u_{k}-[T((j-i) \delta)-I] \sum_{k=1}^{i} u_{k}\right\| \leq 2 \widetilde{M} \int_{i \delta}^{j \delta} \gamma_{Q}(s) d s
$$

for all $1 \leq i+1 \leq j \leq n$. We prove that the set $W$ formed by the functions $z$ defined by (2.22) is relatively compact in $C(I ; X)$. In fact, since $h$ is a fixed function if we denote $\tilde{z}=z-h$, we must prove that $W_{0}=\{\tilde{z}: z \in W\}$ is relatively compact.

First, turning to apply (2.23) and Lemma 2.4, we infer that

$$
\tilde{z}(t)=\int_{0}^{t} T(t-s) f(s, \Psi(s)) d s+\delta \sum_{k=1}^{i-1} u_{k}+\left(t-t_{i-1}\right) u_{i} \in V+U \subseteq U_{1}
$$

for every $z \in W$ and $t \in I$.

Now, we prove that $W_{0}$ is equicontinuous. Let $0 \leq t^{\prime} \leq t \leq \tau$. From (2.23), we can write

$$
\begin{aligned}
\tilde{z}(t)- & \tilde{z}\left(t^{\prime}\right) \\
= & \int_{0}^{t} T(t-s) f(s, \Psi(s)) d s-\int_{0}^{t^{\prime}} T\left(t^{\prime}-s\right) f(s, \Psi(s)) d s+\int_{t^{\prime}}^{t} u(s) d s \\
= & {\left[T\left(t-t^{\prime}\right)-I\right] \int_{0}^{t^{\prime}} T\left(t^{\prime}-s\right) f(s, \Psi(s)) d s+\int_{t^{\prime}}^{t} T(t-s) f(s, \Psi(s)) d s } \\
& +\int_{t^{\prime}}^{t} u(s) d s \\
= & {\left[T\left(t-t^{\prime}\right)-I\right]\left(\tilde{z}\left(t^{\prime}\right)-\int_{0}^{t^{\prime}} u(s) d s\right)+\int_{t^{\prime}}^{t} T(t-s) f(s, \Psi(s)) d s } \\
& +\int_{t^{\prime}}^{t} u(s) d s .
\end{aligned}
$$

In view of that $V$ is a compact set and $\tilde{z}\left(t^{\prime}\right)-\int_{0}^{t^{\prime}} u(s) d s \in V$, in order to prove the assertion, it is sufficient to show that $\int_{t^{\prime}}^{t} u(s) d s$ converges towards zero as $t-t^{\prime} \rightarrow 0$, independent of the construction of $z$.

Since the set $U$ is compact and $\gamma_{Q}$ is integrable for $\varepsilon>0$, there is $\eta_{0}>0$ such that $\|(T(s)-I) u\| \leq \varepsilon / 2$ for all $0 \leq s \leq \eta_{0}, u \in U$, and $2 \widetilde{M} \int_{s^{\prime}}^{s} \gamma_{Q}(s) d s \leq \varepsilon / 2$, for all $s^{\prime}, s \in I,\left|s-s^{\prime}\right| \leq \eta_{0}$. We denote by $C_{1}$ the constant

$$
C_{1}=\sup \left\{\frac{1}{s}\|(T(s)-I) u\|: \eta_{0} \leq s \leq \tau, u \in U\right\}
$$

and we take $0<\eta \leq \min \left\{\eta_{0}, \varepsilon \eta_{0} / 2 N_{1}, \varepsilon / 2 C_{1}\right\}$. 
Initially, we assume that $t^{\prime}$ and $t$ coincide with some points of the division. Thus, we suppose that $t^{\prime}=t_{i}$ and $t=t_{j}$. In this case, we have that

$$
\begin{aligned}
\int_{t^{\prime}}^{t} u(s) d s= & \delta \sum_{k=i+1}^{j} u_{k} \\
= & \delta \sum_{k=i+1}^{j} u_{k}-[T((j-i) \delta)-I] \delta \sum_{k=1}^{i} u_{k} \\
& +[T((j-i) \delta)-I] \delta \sum_{k=1}^{i} u_{k},
\end{aligned}
$$

and applying (2.25), we obtain the estimation

$$
\left\|\int_{t^{\prime}}^{t} u(s) d s\right\| \leq 2 \widetilde{M} \int_{t^{\prime}}^{t} \gamma_{Q}(s) d s+\left\|\left[T\left(t-t^{\prime}\right)-I\right] \delta \sum_{k=1}^{i} u_{k}\right\|
$$

Consequently, since $\delta \sum_{k=1}^{i} u_{k} \in U$, if $t_{j}-t_{i} \leq \eta_{0}$, it follows that $\left\|\int_{t^{\prime}}^{t} u(s) d s\right\| \leq \varepsilon$.

Let $t-t^{\prime} \leq \eta$. Regarding the relative location of points $t_{k}$, we analyze three possible situations. In first case, we assume that there is no point $t_{k}$ between $t^{\prime}$ and $t$. Hence, there is an index $i$ such that $t_{i}<t^{\prime}<t \leq t_{i+1}$ and $\int_{t^{\prime}}^{t} u(s) d s=$ $\left(t-t^{\prime}\right) u_{i+1}$. From (2.25), we have that

$$
\left\|u_{i+1}\right\| \leq \frac{2 \widetilde{M}}{\delta} \int_{t_{i}}^{t_{i+1}} \gamma_{Q}(s) d s+\left\|(T(\delta)-I) \sum_{k=1}^{i} u_{k}\right\|
$$

Hence, if $\delta \leq \eta_{0}$, using that $t-t^{\prime} \leq \delta$ from the above expression, it follows that

$$
\left(t-t^{\prime}\right)\left\|u_{i+1}\right\| \leq \frac{\varepsilon}{2}+\frac{\left(t-t^{\prime}\right)}{\delta}\left\|(T(\delta)-I) \delta \sum_{k=1}^{i} u_{k}\right\| \leq \varepsilon,
$$

while if $\delta \geq \eta_{0}$, we obtain that

$$
\left(t-t^{\prime}\right)\left\|u_{i+1}\right\| \leq\left(t-t^{\prime}\right) \frac{N_{1}}{\eta_{0}}+\left(t-t^{\prime}\right) \frac{1}{\delta}\left\|(T(\delta)-I) \delta \sum_{k=1}^{i} u_{k}\right\| \leq \varepsilon
$$

which establishes the assertion in this case.

Now, we assume that there is an index $i$ such that $t_{i-1}<t^{\prime}<t_{i}<t<t_{i+1}$. Therefore, from our definitions, it follows that

$$
\int_{t^{\prime}}^{t} u(s) d s=\left(t_{i}-t^{\prime}\right) u_{i}+\left(t-t_{i}\right) u_{i+1}
$$

Since $t_{i}-t^{\prime}<t-t^{\prime}$ and $t-t_{i}<t-t^{\prime}$, we can argue as in the preceding case. 
Finally, we consider that there are indices $i<j$ such that $t_{i-1}<t^{\prime}<t_{i}<t_{j}<$ $t<t_{j+1}$. Clearly,

$$
\left\|v(t)-v\left(t^{\prime}\right)\right\| \leq\left\|v(t)-v\left(t_{j}\right)\right\|+\left\|v\left(t_{j}\right)-v\left(t_{i}\right)\right\|+\left\|v\left(t_{i}\right)-v\left(t^{\prime}\right)\right\|,
$$

where we have abbreviated $v(t)=\int_{0}^{t} u(s) d s$. In view of that $t_{j}-t_{i} \leq t-t^{\prime}$, from our initial remark, we obtain that $\left\|v\left(t_{j}\right)-v\left(t_{i}\right)\right\| \leq \varepsilon$. Thus, this case is reduced to estimate the first and third term on the right-hand side of the above expression. For the first term, we observe that $v(t)$ is a convex combination of $v\left(t_{j}\right)$ and $v\left(t_{j+1}\right)$. Therefore,

$$
\left\|v(t)-v\left(t_{j}\right)\right\| \leq\left\|v\left(t_{j+1}\right)-v\left(t_{j}\right)\right\|,
$$

and since $\delta=t_{j+1}-t_{j} \leq t-t^{\prime}$, we can repeat the previous argument. The third term is estimated similarly.

From the Ascoli-Arzela theorem, it follows that $W_{0}$ is relatively compact and so is $W=h+W_{0}$.

STEP 3. Now let $\varepsilon>0$ be fixed. Without loss of generality, we assume also that $\varepsilon \leq \min \left\{\tau / 2,2 N_{1}\right\}$ and we take $\varepsilon_{1}=\varepsilon / 2 \widetilde{M} \tau$. Using the compactness of sets $\mathscr{S}$ and $W$ as well as the continuity of $f$, we infer the existence of $0<\delta_{1} \leq 2 \mathrm{K \varepsilon}$ such that

$$
\left\|f\left(s, \psi_{1}\right)-f\left(s, \psi_{2}\right)\right\| \leq \varepsilon_{1}
$$

for all $s \in I$ and for every $\psi_{1}, \psi_{2} \in H(W \cup \mathscr{S})(I)$ such that $\left\|\psi_{1}-\psi_{2}\right\|_{\mathscr{B}} \leq \delta_{1}$. Similarly, there is $\delta_{2}>0$ such that

$$
\|x(s)-x(t)\| \leq \frac{\delta_{1}}{4 K}
$$

for all $x(\cdot) \in W \cup \mathscr{Y}$ and $s, t \in I$ such that $|t-s| \leq \delta_{2}$.

Now, we choose $\delta=\tau / n \leq \min \left\{\delta_{2}, \delta_{1} \tau / 2 K \varepsilon\right\}$.

In the sequel, we consider the division $d$ defined by $t_{i}=i \delta, i=0, \ldots, n$. Let $W_{\varepsilon}$ be the set formed by the functions defined by (2.22) with $\left(u_{1}, \ldots, u_{n}\right) \in Z_{\varepsilon}$, where $Z_{\varepsilon}$ is the set consisting of points $\left(u_{1}, \ldots, u_{n}\right) \in((2 / \delta) U)^{n}$ that satisfy the following conditions:

(i) $\delta \sum_{k=1}^{i} u_{k} \in U$,

(ii) $\delta\left\|\sum_{k=1}^{i} u_{k}\right\| \leq \widetilde{M} t_{i} \varepsilon_{1}$,

(iii) $\left\|\delta \sum_{k=i+1}^{j} u_{k}-\delta[T((j-i) \delta)-I] \sum_{k=1}^{i} u_{k}\right\| \leq 2 \widetilde{M} \int_{t_{i}}^{t_{j}} \gamma_{Q}(s) d s$, for all $i=1, \ldots, n$ and $j \geq i+1$.

We notice that condition (ii) implies that $\delta\left\|\sum_{k=1}^{i} u_{k}\right\| \leq N_{1}$. Next, we establish some properties of $W_{\varepsilon}$. 
STEP 4. The set $W_{\varepsilon}$ is connected. This assertion is an easy consequence of the fact that the functions $z \in W_{\varepsilon}$ depend continuously on the choice of $\left(u_{1}, \ldots, u_{n}\right) \in Z_{\varepsilon}$ and $Z_{\varepsilon}$ is convex by our construction.

STEP 5. In this step, we show that the solutions of (2.3) can be approximated by the elements in $W_{\varepsilon}$. Let $x \in \mathscr{S}$ be fixed. We proceed to define $z \in W_{\varepsilon}$ so that $\|x-z\|_{\infty} \leq \varepsilon$. We define $z(\cdot)$ inductively on the intervals $\left[t_{i-1}, t_{i}\right]$.

Let $i=1$. In this case, $t_{1}=\delta$ and we take

$$
u_{1}=\frac{1}{t_{1}} \int_{0}^{t_{1}} T\left(t_{1}-s\right)\left[f\left(s, x_{s}\right)-f(s, \varphi)\right] d s .
$$

It is clear from our construction that $u_{1} \in(1 / \delta) U$. Moreover, from (2.38), it follows that $\left\|x_{s}-\varphi\right\|_{\mathscr{B}} \leq \delta_{1} / 4$ so that (2.37) implies that $\left\|f\left(s, x_{s}\right)-f(s, \varphi)\right\| \leq$ $\varepsilon_{1}$ for all $0 \leq s \leq \delta$ and this yields that $\left\|u_{1}\right\| \leq \widetilde{M} \varepsilon_{1}$. We define

$$
z(t)=h(t)+\int_{0}^{t} T(t-s) f(s, \varphi) d s+t u_{1}
$$

for $0 \leq t \leq t_{1}$. From this expression, it follows that

$$
\begin{aligned}
z\left(t_{1}\right) & =h\left(t_{1}\right)+\int_{0}^{t_{1}} T\left(t_{1}-s\right) f(s, \varphi) d s+t_{1} u_{1} \\
& =h\left(t_{1}\right)+\int_{0}^{t_{1}} T\left(t_{1}-s\right) f(s, \varphi) d s=x\left(t_{1}\right) .
\end{aligned}
$$

Moreover, for $0<t \leq t_{1}$, we have

$$
\begin{aligned}
\|x(t)-z(t)\| & \leq\left\|\int_{0}^{t} T(t-s)\left[f\left(s, x_{s}\right)-f(s, \varphi)\right] d s\right\|+t\left\|u_{1}\right\| \\
& \leq 2 \widetilde{M} \varepsilon_{1} t \leq \frac{\delta_{1}}{2 K} .
\end{aligned}
$$

Proceeding by induction, we assume now that we have selected the elements $u_{k}, k=1, \ldots, i-1$ such that $\left(u_{1}, \ldots, u_{i-1}, 0, \ldots, 0\right) \in Z_{\varepsilon}$ and the function $z(t)$ given by (2.22) for $t \in\left[0, t_{i-1}\right]$ satisfies $z\left(t_{k}\right)=x\left(t_{k}\right)$ and the estimation

$$
\|x(t)-z(t)\| \leq \frac{\delta_{1}}{2 K}, \quad 0 \leq t \leq t_{i-1} .
$$

We define now the function $z$ on $\left[t_{i-1}, t_{i}\right]$. We begin by selecting

$$
\begin{aligned}
u_{i}= & \frac{1}{\delta} \sum_{k=1}^{i-1} \int_{t_{k-1}}^{t_{k}}\left[T\left(t_{i}-s\right)-T\left(t_{i-1}-s\right)\right]\left[f\left(s, x_{s}\right)-f\left(s, z_{t_{k-1}}\right)\right] d s \\
& +\frac{1}{\delta} \int_{t_{i-1}}^{t_{i}} T\left(t_{i}-s\right)\left[f\left(s, x_{s}\right)-f\left(s, z_{t_{i-1}}\right)\right] d s .
\end{aligned}
$$


Utilizing the function $\Psi(\cdot)$ defined previously, we can abbreviate

$$
\begin{aligned}
u_{i}= & \frac{1}{\delta} \int_{0}^{t_{i}} T\left(t_{i}-s\right)\left[f\left(s, x_{s}\right)-f(s, \Psi(s))\right] d s \\
& -\frac{1}{\delta} \int_{0}^{t_{i-1}} T\left(t_{i-1}-s\right)\left[f\left(s, x_{s}\right)-f(s, \Psi(s))\right] d s .
\end{aligned}
$$

Initially, we establish that $\left(u_{1}, \ldots, u_{i}, 0, \ldots, 0\right) \in Z_{\varepsilon}$. From the above expression, it follows easily that $\delta u_{i} \in 2 U$ and

$$
\delta \sum_{k=1}^{i} u_{k}=\int_{0}^{t_{i}} T\left(t_{i}-s\right)\left[f\left(s, x_{s}\right)-f(s, \Psi(s))\right] d s,
$$

which implies that $\delta \sum_{k=1}^{i} u_{k} \in U$ and $\delta\left\|\sum_{k=1}^{i} u_{k}\right\| \leq N_{1}$. Moreover, for $m+1 \leq$ $j \leq i$, we have that

$$
\begin{aligned}
\delta \sum_{k=m+1}^{j} u_{k}= & \delta \sum_{k=1}^{j} u_{k}-\delta \sum_{k=1}^{m} u_{k} \\
= & \int_{0}^{t_{j}} T\left(t_{j}-s\right)\left[f\left(s, x_{s}\right)-f(s, \Psi(s))\right] d s \\
& -\int_{0}^{t_{m}} T\left(t_{m}-s\right)\left[f\left(s, x_{s}\right)-f(s, \Psi(s))\right] d s \\
= & {\left[T\left(t_{j}-t_{m}\right)-I\right] \int_{0}^{t_{m}} T\left(t_{m}-s\right)\left[f\left(s, x_{s}\right)-f(s, \Psi(s))\right] d s } \\
& +\int_{t_{m}}^{t_{j}} T\left(t_{j}-s\right)\left[f\left(s, x_{s}\right)-f(s, \Psi(s))\right] d s
\end{aligned}
$$

which yields

$$
\begin{aligned}
& \delta \sum_{k=m+1}^{j} u_{k}-\left[T\left(t_{j}-t_{m}\right)-I\right] \delta \sum_{k=1}^{m} u_{k} \\
& \quad=\int_{t_{m}}^{t_{j}} T\left(t_{j}-s\right)\left[f\left(s, x_{s}\right)-f(s, \Psi(s))\right] d s
\end{aligned}
$$

which in turn implies the estimation

$$
\left\|\delta \sum_{k=m+1}^{j} u_{k}-[T((j-m) \delta)-I] \delta \sum_{k=1}^{m} u_{k}\right\| \leq 2 \widetilde{M} \int_{t_{m}}^{t_{j}} \gamma_{Q}(s) d s .
$$

In addition, for $t_{i-1}<s \leq t_{i}$, we can write

$$
x_{s}-\Psi(s)=x_{s}-x_{t_{i-1}}+x_{t_{i-1}}-z_{t_{i-1}} .
$$

From (2.38), we have that $\left\|x_{s}-x_{t_{i-1}}\right\|_{\circledast} \leq \delta_{1} / 4$ and, by induction, $\| x_{t_{i-1}}-$ $z_{t_{i-1}} \|_{\mathscr{B}} \leq \delta_{1} / 2$. Combining these estimations with (2.37), we infer that $\delta\left\|\sum_{k=1}^{i} u_{k}\right\| \leq \widetilde{M} t_{i} \varepsilon_{1}$, which establishes that $\left(u_{1}, \ldots, u_{i}, 0, \ldots, 0\right) \in Z_{\varepsilon}$. 
Now, we define $z(t)$ for $t_{i-1}<t \leq t_{i}$ by means of formula (2.22). Using this expression as well as the choice of $u_{k}, k=1, \ldots, i$, we infer that

$$
\begin{aligned}
x\left(t_{i}\right)-z\left(t_{i}\right)= & \int_{0}^{t_{i}} T\left(t_{i}-s\right)\left[f\left(s, x_{s}\right)-f(s, \Psi(s))\right] d s-\delta \sum_{k=1}^{i} u_{k} \\
= & \int_{0}^{t_{i}} T\left(t_{i}-s\right)\left[f\left(s, x_{s}\right)-f(s, \Psi(s))\right] d s \\
& -\int_{0}^{t_{i-1}} T\left(t_{i-1}-s\right)\left[f\left(s, x_{s}\right)-f(s, \Psi(s))\right] d s-\delta u_{i} \\
= & 0 .
\end{aligned}
$$

Moreover, from (2.38) and the choice of $\delta$, it follows that

$$
\|x(t)-z(t)\| \leq\left\|x(t)-x\left(t_{i-1}\right)\right\|+\left\|z(t)-z\left(t_{i-1}\right)\right\| \leq \frac{\delta_{1}}{2 K},
$$

which establishes the assertion.

STEP 6. In this step, we prove that the elements of $W_{\varepsilon}$ are approximate solutions of (2.3). Specifically, we show that

$$
\left\|z(t)-h(t)-\int_{0}^{t} T(t-s) f\left(s, z_{s}\right) d s\right\| \leq \varepsilon
$$

for all $t \in I$ and $z \in W_{\varepsilon}$.

In fact, for $t_{i-1}<t \leq t_{i}$, using (2.22), we have

$$
\begin{aligned}
z(t)- & h(t)-\int_{0}^{t} T(t-s) f\left(s, z_{s}\right) d s \\
= & \int_{0}^{t} T(t-s)\left[f(s, \Psi(s))-f\left(s, z_{s}\right)\right] d s \\
& +\delta \sum_{k=1}^{i-1} u_{k}+\left(t-t_{i-1}\right) u_{i}
\end{aligned}
$$

and employing now (2.37), (2.38), and the choice of $\delta$, we can establish the estimation

$$
\begin{aligned}
\| z(t) & -h(t)-\int_{0}^{t} T(t-s) f\left(s, z_{s}\right) d s \| \\
\leq & \left\|\int_{0}^{t} T(t-s)\left[f(s, \Psi(s))-f\left(s, z_{s}\right)\right] d s\right\| \\
& +\left\|\delta \sum_{k=1}^{i-1} u_{k}+\left(t-t_{i-1}\right) u_{i}\right\| \\
\leq & 2 \widetilde{M} \tau \varepsilon_{1},
\end{aligned}
$$

which shows our assertion. 
Since $\mathscr{S}$ is the set of fixed points of the map $\mathscr{T}$ given by (2.4), gathering Step 1 and Step 6 and applying Corollary 1.2, we obtain that $\mathscr{Y}$ is connected in the space $C(I ; X)$. Finally, to complete the proof, we observe that the map $\Lambda: C_{\varphi} \rightarrow C(I ; \mathscr{B}), x(\cdot) \rightarrow\left(t \rightarrow x_{t}\right)$, is continuous and that $H(\mathscr{Y})=\Lambda(\mathscr{Y})$.

3. Application. The equations studied in this work are widely used to model concrete problems. We consider, as an example, the equation

$$
\begin{gathered}
\frac{\partial u(\xi, t)}{\partial t}=d \frac{\partial^{2} u(\xi, t)}{\partial \xi^{2}}+h\left(\int_{0}^{\infty} q(s) u(\xi, t-s) d s\right), \quad 0<\xi<1, t>0 \\
u(\xi, t)=0, \quad \xi=0,1
\end{gathered}
$$

where $d$ is a positive constant and $q(\cdot)$ and $h$ are continuous functions that satisfy some conditions that will be specified later, which is a simplification of the predator-prey model proposed by Cohen et al. [2] to represent some inherent time delay in the population model.

To represent this problem in an abstract frame, we take $X:=L^{2}([0,1])$ and $x(t):=u(\cdot, t)$. The operator $A$ is given by

$$
A u(\xi)=d u^{\prime \prime}(\xi)
$$

with domain

$$
D(A):=\left\{u(\cdot) \in L^{2}([0,1]): u^{\prime \prime}(\cdot) \in L^{2}([0, \pi]), u(0)=u(1)=0\right\}
$$

It is well known that $A$ generates a strongly continuous semigroup $T(\cdot)$ which is compact, analytic, and selfadjoint. Furthermore, $A$ has discrete spectrum with simple eigenvalues $-n^{2} \pi^{2} d, n \in \mathbb{N}$. The set of normalized eigenvectors is complete in $X$, which shows that $\|T(t)\| \leq e^{-d \pi^{2} t}, t \geq 0$, so that $\widetilde{M}=1$.

Let $\mathscr{B}$ denote the space $U C_{g}$ in the terminology of [8]. Here, $g$ is a positive continuous function defined in $(-\infty, 0]$ which satisfies conditions (g2) and (g4) in [8] and the function $G(t)=\sup _{-\infty<\theta \leq-t}(g(t+\theta) / g(\theta)) \rightarrow 0$, as $t \rightarrow \infty$. In this case, $\mathscr{B}$ is a fading memory space (see [8, Theorem 1.3.8, Example 7.1.7]) and $H=g(0), K(t)=\sup _{-t \leq \theta \leq 0} 1 / g(\theta)$, and $M(t)=G(t)$.

Assuming that

$$
C=\int_{-\infty}^{0} g(\theta)|q(-\theta)| d \theta<\infty,
$$

it follows that the Bochner's integral

$$
F(\varphi)=\int_{-\infty}^{0} q(-\theta) \varphi(\theta) d \theta
$$


defines a bounded linear operator $F: \mathscr{B} \rightarrow X$. Hence, we have that

$$
F(\varphi)(\xi)=\int_{-\infty}^{0} q(-\theta) \varphi(\xi, \theta) d \theta \text { a.e., }
$$

where we have abbreviated the notation by writing $\varphi(\xi, \theta)$ instead of $\varphi(\theta)(\xi)$, $\theta \in(-\infty, 0]$, and $\xi \in[0,1]$.

We assume in addition that there are constants $C_{1} \geq 0$ and $0 \leq \alpha \leq 1$ so that $h$ satisfies the condition $|h(t)| \leq C_{1}|t|^{\alpha}, t \in \mathbb{R}$. We define $f(t, \varphi)(\xi)=$ $h(F(\varphi)(\xi)), 0 \leq \xi \leq 1$.

With these definitions, problem (3.1) can be set in the form (1.1) and (1.2) and we can verify easily that the hypotheses of Theorem 2.2 hold. In fact, it is not difficult to see that $f$ satisfies the Carathéodory conditions. Furthermore,

$$
\|f(t, \varphi)\|_{2} \leq C_{1} C^{\alpha}\|\varphi\|_{\mathscr{B}}^{\alpha}
$$

which shows that condition (H1) holds with $\gamma_{R}(t)=C_{1} C^{\alpha} R^{\alpha}$, and, in turn, this definition shows that (H4) and (H6) are fulfilled for all $\tau>0$ when $\alpha<1$, while (H4) and (H6) are verified for $\tau>0$ small enough when $\alpha=1$. Finally, condition (H2) follows from the fact that $T$ is a compact semigroup . Consequently, the hypotheses of Theorems 2.2 and 2.5 are satisfied and we can assert that problem (3.1) has mild solutions and that the set formed by these solutions is connected.

It is worth to point out that we have obtained these properties without assuming that $h$ satisfies a local Lipschitz condition.

ACKNOWLEDgment. This work was supported by DICYT-USACH Project 04-0133HM and FONDECYT Project 1020259.

\section{REFERENCES}

[1] T. Cazenave and A. Haraux, An Introduction to Semilinear Evolution Equations, Oxford Lecture Series in Mathematics and Its Applications, vol. 13, Oxford University Press, New York, 1998.

[2] D. S. Cohen, P. S. Hagan, and H. C. Simpson, Spatial structures in predator-prey communities with hereditary effects and diffusion, Math. Biosci. 44 (1979), no. 3-4, 167-177.

[3] K.-J. Engel and R. Nagel, One-Parameter Semigroups for Linear Evolution Equations, Graduate Texts in Mathematics, vol. 194, Springer-Verlag, New York, 2000.

[4] J. K. Hale and J. Kato, Phase space for retarded equations with infinite delay, Funkcial. Ekvac. 21 (1978), no. 1, 11-41.

[5] P. Hartman, Ordinary Differential Equations, John Wiley \& Sons, New York, 1964.

[6] H. R. Henríquez, Periodic solutions of quasi-linear partial functional-differential equations with unbounded delay, Funkcial. Ekvac. 37 (1994), no. 2, 329343.

[7] _ Approximation of abstract functional-differential equations with unbounded delay, Indian J. Pure Appl. Math. 27 (1996), no. 4, 357-386. 
[8] Y. Hino, S. Murakami, and T. Naito, Functional-Differential Equations with Infinite Delay, Lecture Notes in Mathematics, vol. 1473, Springer-Verlag, Berlin, 1991.

[9] T. Kaminogo, Kneser's property and boundary value problems for some retarded functional differential equations, Tôhoku Math. J. (2) 30 (1978), no. 3, 471486.

[10] C.-M. Marle, Mesures et Probabilités, Collection Enseignement des Sciences, no. 19, Hermann, Paris, 1974 (French).

[11] I. Miyadera, Nonlinear Semigroups, Translations of Mathematical Monographs, vol. 109, American Mathematical Society, Rhode Island, 1992.

[12] A. Pazy, Semigroups of Linear Operators and Applications to Partial Differential Equations, Applied Mathematical Sciences, vol. 44, Springer-Verlag, New York, 1983.

[13] E. Zeidler, Nonlinear Functional Analysis and Its Applications. I, Springer-Verlag, New York, 1986.

Hernán R. Henríquez: Departamento de Matemática, Universidad de Santiago, Casilla 307, Correo 2, Santiago, Chile

E-mail address: hhenriqu@1auca.usach.c1 


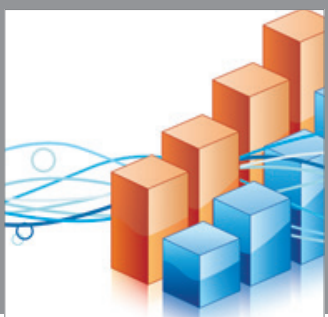

Advances in

Operations Research

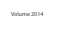

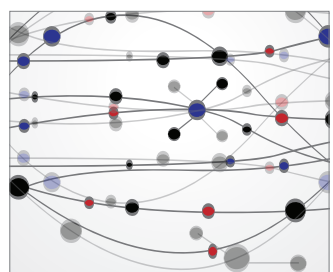

\section{The Scientific} World Journal
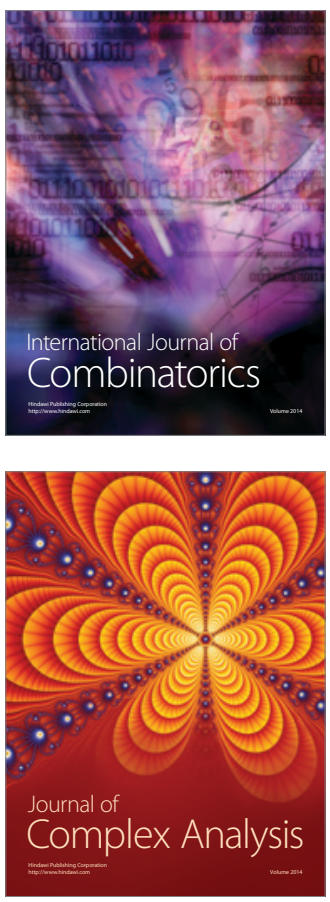

International Journal of

Mathematics and

Mathematical

Sciences
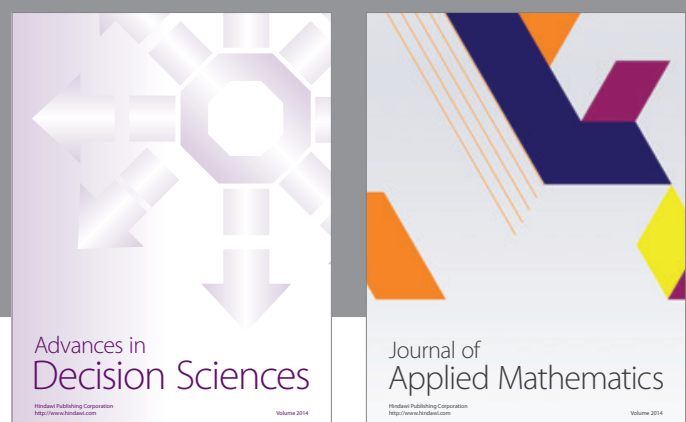

Journal of

Applied Mathematics
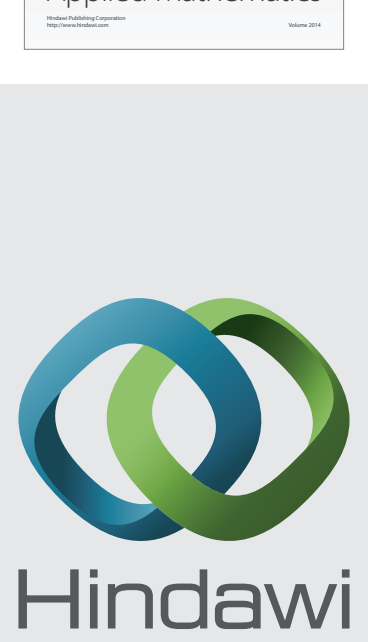

Submit your manuscripts at http://www.hindawi.com
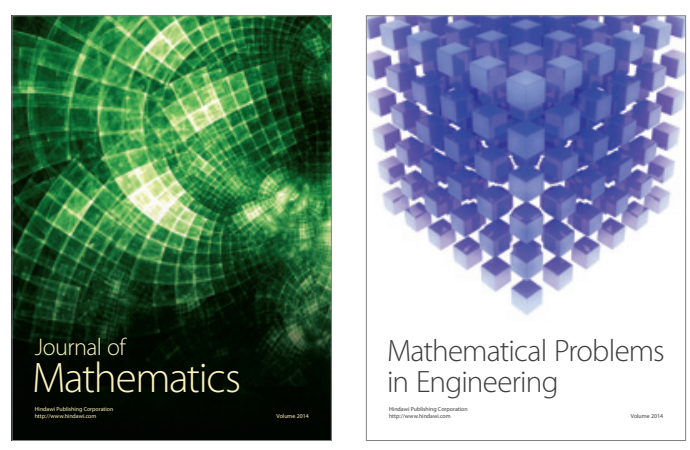

Mathematical Problems in Engineering
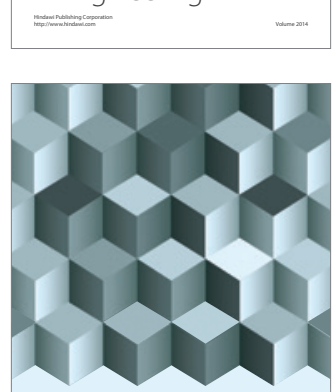

Journal of

Function Spaces
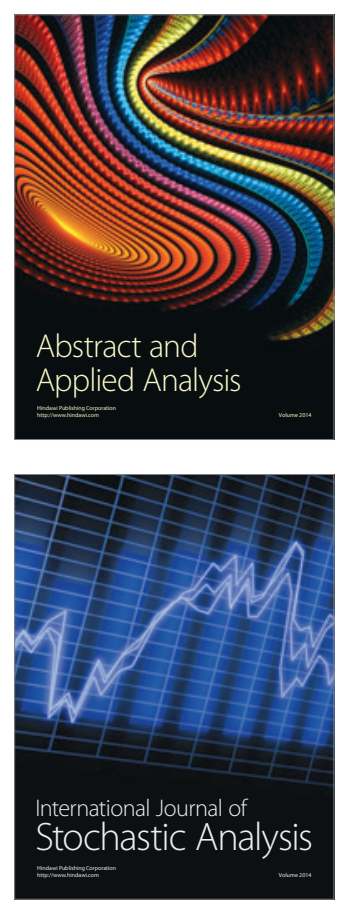

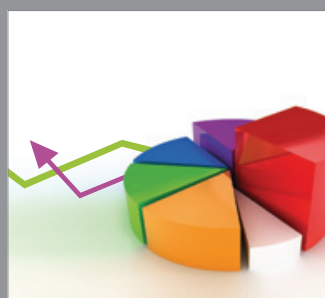

ournal of

Probability and Statistics

Promensencen
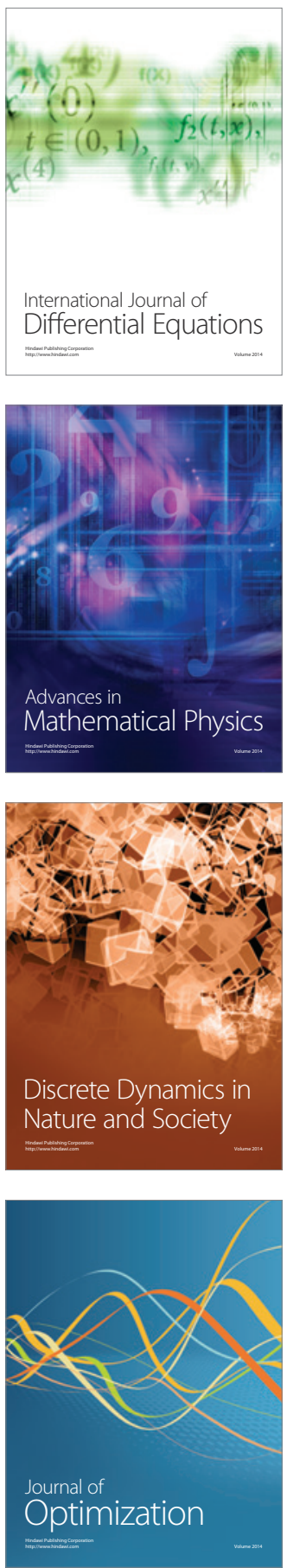